\title{
T2 mapping of lumbosacral nerves in patients suffering from unilateral radicular pain due to degenerative disc disease
}

\author{
Nico Sollmann, MD, PhD, ${ }^{1-3}$ Dominik Weidlich, MSc, ${ }^{4}$ Barbara Cervantes, $\mathrm{PhD},{ }^{4}$ \\ Elisabeth Klupp, MD, ${ }^{1}$ Carl Ganter, PhD, ${ }^{4}$ Hendrik Kooijman, PhD, ${ }^{5}$ Claus Zimmer, MD, ${ }^{1,2}$ \\ Ernst J. Rummeny, MD, ${ }^{4}$ Bernhard Meyer, MD, ${ }^{3}$ Thomas Baum, MD, Jan S. Kirschke, MD, ${ }^{1}$ and \\ Dimitrios C. Karampinos, $\mathrm{PhD}^{4}$
}

\begin{abstract}
${ }^{1}$ Department of Diagnostic and Interventional Neuroradiology, ${ }^{2}$ TUM-Neuroimaging Center, ${ }^{3}$ Department of Neurosurgery, and ${ }^{4}$ Department of Diagnostic and Interventional Radiology, Klinikum rechts der Isar, Technische Universität München, Munich; and ${ }^{5}$ Philips Healthcare, Hamburg, Germany
\end{abstract}

\begin{abstract}
OBJECTIVE Lumbosacral radicular syndrome (LRS) is a very common condition, often requiring diagnostic imaging with the aim of elucidating a structural cause when symptoms are longer lasting. However, findings on conventional anatomical MRI do not necessarily correlate with clinical symptoms, and it is primarily performed for the qualitative evaluation of surrounding compressive structures, such as herniated discs, instead of to evaluate the nerves directly. The present study investigated the performance of quantitative imaging by using magnetic resonance neurography (MRN) in patients with LRS.
\end{abstract}

METHODS Eighteen patients (55.6\% males, mean age $64.4 \pm 10.2$ years), with strict unilateral LRS matching at least one dermatome and suspected disc herniation, underwent high-resolution 3-T MRN using T2 mapping. On T2 maps, the presumably affected and contralateral unaffected nerves were identified; subsequent regions of interest (ROIs) were placed at preganglionic, ganglionic, and postganglionic sites; and T2 values were extracted. Patients then underwent an epidural steroid injection (ESI) with local anesthetic agents at the site of suspected nerve affection. T2 values of the affected nerves were compared against the contralateral nerves. Furthermore, receiver operating characteristics were calculated based on the measured T2 values and the responsiveness to ESI.

RESULTS The mean T2 value was $77.3 \pm 1.9 \mathrm{msec}$ for affected nerves and $74.8 \pm 1.4 \mathrm{msec}$ for contralateral nerves ( $p$ $<0.0001$ ). In relation to ESI performed at the site of suspected nerve affection, MRN with T2 mapping had a sensitivity/ specificity of $76.9 \% / 60.0 \%$ and a positive/negative predictive value of $83.3 \% / 50.0 \%$. Signal alterations in affected nerves according to qualitative visual inspection were present in only $22.2 \%$ of patients.

CONCLUSIONS As one of the first of its kind, this study revealed elevated T2 values in patients suffering from LRS. T2 values of lumbosacral nerves might be used as more objective parameters to directly detect nerve affection in such patients.

https://thejns.org/doi/abs/10.3171/2018.10.SPINE181172

KEYWORDS degenerative disc disease; epidural steroid injection; lumbar; lumbosacral plexus; lumbosacral radicular syndrome; magnetic resonance neurography; sacral; T2 mapping

$\mathrm{L}$ ow back pain (LBP) is a very common condition that is known to be among the leading causes of years lived with disability, entailing significant decreases (ife. . $^{13,19,41,43}$ Aside from perceiving pain focally in the lower back, patients can suffer from additional or isolated lumbosacral radicular syndrome (LRS), which is commonly referred to as a radiating pain, perceived in one or more lumbar or sacral dermatomes that may be accompanied by other radicular irritation symptoms in addition to the pain, such as paresthesia or impairment of motor function..$^{14,40}$ LRS shows a high prevalence of $4.6 \%-13.4 \%$ and a high lifetime prevalence of $1.2 \%-43 \% .^{28,40} \mathrm{LRS}$ is

ABBREVIATIONS BMRC = British Medical Research Council; ESI = epidural steroid injection; FN = false negative; FOV = field of view; FP = false positive; LBP = low back pain; LRS = lumbosacral radicular syndrome; $L S P=$ lumbosacral plexus; $M R N=$ magnetic resonance neurography; NPV = negative predictive value; $P P V=$ positive predictive value; $\mathrm{ROC}$ = receiver operating characteristic; $\mathrm{ROI}$ = region of interest; $\mathrm{TN}=$ true negative; $\mathrm{TP}$ = true positive; TSE = turbo spin echo; VAS = visual analog scale. SUBMITTED September 20, 2018. ACCEPTED October 30, 2018.

INCLUDE WHEN CITING Published online February 22, 2019; DOI: 10.3171/2018.10.SPINE181172. 
most often suspected to be caused by compression of the nerve root by degenerative disc material.

In the course of severe or longer-lasting LRS with or without LBP, imaging methods, including CT and anatomical MRI, are routinely applied in an attempt to identify structural pathologies that might be responsible for nerve compression and thus the pain condition. In cases in which multisegmental structural pathologies are present or symptoms are not clearly associated with one nerve root only, selective segmental nerve blocks by epidural steroid injections (ESIs) and local anesthetics may be helpful in detecting and treating the particular site most probably causing LRS..$^{40,42}$

Regarding imaging, MRI is often preferred over CT because of the absence of radiation exposure and a better visualization of soft tissues, thus enhancing the visual detection of herniated discs. ${ }^{26,40}$ However, it is well known that imaging findings do not necessarily correlate with clinical symptoms. In previous investigations, herniated discs were identified by CT or MRI in about $20 \%-36 \%$ of asymptomatic subjects, and symptoms can even resolve under conservative treatment without a detectable volume decrease of the herniated disc in a considerable number of patients. ${ }^{12,20,31}$

Magnetic resonance neurography (MRN) has gained increasing importance as a novel add-on diagnostic tool that enables the identification and characterization of various nerve pathologies. ${ }^{79}$ When performed with advanced high-resolution sequences, MRN can even depict the small and highly oblique nerves of the brachial plexus or lumbosacral plexus (LSP), which are known to be particularly challenging for imaging., ${ }^{4}, 10,11,21,36$ However, most previous work has relied on qualitative MRN evaluations with a focus on the assessments of signal alterations on T2-weighted images, although MRN principally also offers the possibility for quantitative evaluation., ${ }^{4,10,11,21,36} \mathrm{In}$ this context, evaluation of nerves only based on qualitative parameters remains challenging and is regarded as subjective; in contrast, quantitative methods, such as T2 mapping, may reflect a more objective approach. Recent studies have successfully applied T2 mapping of the LSP in healthy subjects, with the aim of providing normative T2 values and evaluating reliability. ${ }^{29,37}$ However, research on quantitative MRN by means of T2 mapping is lacking in patients with LRS, although it might improve imagingbased diagnostics by providing more objective information about the affected nerves themselves instead of primarily enabling qualitative evaluation of compressing surrounding structures, as conventional anatomical MRI does.

In the present study, we applied high-resolution MRN with T2 mapping of LSP nerves in patients suffering from unilateral LRS related to disc herniation to detect affected nerves by means of quantitative imaging and to correlate imaging data with clinical symptoms and the results of ESI.

\section{Methods \\ Ethics}

The study protocol was approved by the local ethics commission and was followed in accordance with the
Declaration of Helsinki. Written informed consent was obtained from all patients prior to MRN.

\section{Patients and Study Inclusion}

We applied high-resolution MRN in combination with T2 mapping for the quantitative evaluation of LSP nerves in patients suffering from LRS with or without LBP.

The following inclusion criteria were defined prior to enrollment:

- written informed consent

- age above 18 years

- strict unilateral LRS matching at least one dermatome (according to clinical examination)

- presence of symptoms (pain with or without additional paresthesia or impairment of motor function) for at least 4 weeks and not longer than 6 months

- previous anatomical MRI suggesting disc herniation due to degeneration with unilateral nerve contact of degenerated disc material, and

- scheduled unilateral ESI for diagnostic purposes (at the site of the affected nerve according to clinical examination and previous anatomical MRI findings).

The following exclusion criteria were considered:

- pregnancy

- contraindications to MRI (e.g., cochlear implant)

- contraindications to ESI (e.g., blood coagulation disorders, suspected or confirmed allergy to local anesthetics or corticosteroids)

- previous surgery at the lumbar spine level (except for surgical decompression without any implants)

- previous ESI within 4 weeks prior to MRN scheduled for this study, and

- bilateral LRS or no clear radicular pain (according to clinical examination).

Patients fulfilling the inclusion criteria first underwent further detailed clinical examination, including assessments of coordination, sensory function, and lower-extremity muscle strength according to the British Medical Research Council (BMRC) scale. Furthermore, the Oswestry Disability Index was administered prior to MRN. ${ }^{16,17}$ Overall perception of radicular pain was classified according to the visual analog scale (VAS). ${ }^{30,34}$

\section{MRN}

\section{Image Acquisition}

MRN was performed using a 3-T whole-body MRI scanner (Ingenia, Philips Healthcare) in combination with a 16-channel torso coil array and a posterior 12-channel coil array. The scanning protocol included the following sequences: a flow-suppressed T2-weighted, 3D turbo spin echo (TSE) sequence with an isotropic voxel size of $2 \mathrm{~mm}$ to depict LSP anatomy, with the field of view (FOV) covering at least the LSP nerves L2-S2:4,37 and an adiabatic T2-prepared, 3D TSE sequence with fat suppression using spectral attenuated inversion recovery and variable duration of the T2 preparation for the purpose of T2 mapping of LSP nerves with the following sequence parameters: FOV $38 \times 38 \times 8 \mathrm{~cm}^{3}$; acquisition voxel $2 \times 2 \times 2$ $\mathrm{mm}^{3}$; echo train length 80 ; T2 preparation durations of 
Sagittal survey patient 1

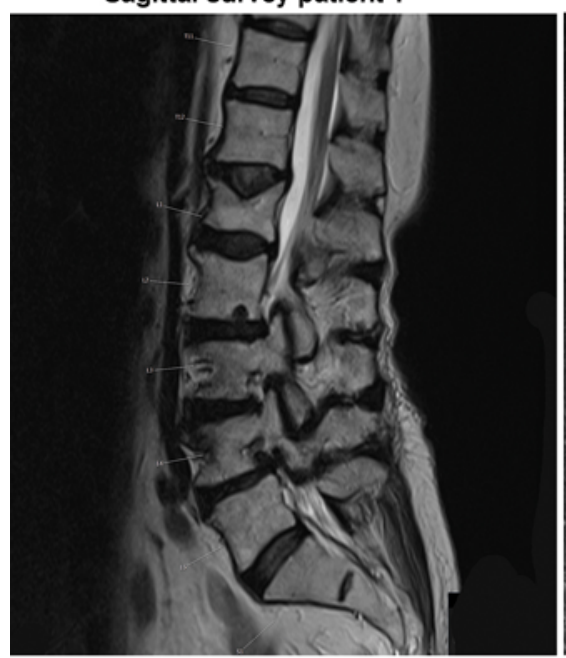

Sagittal survey patient 2

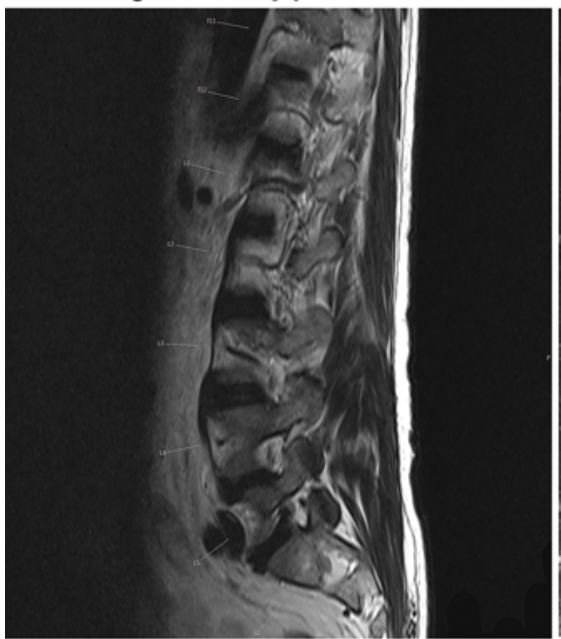

MIP of T2W TSE sequence

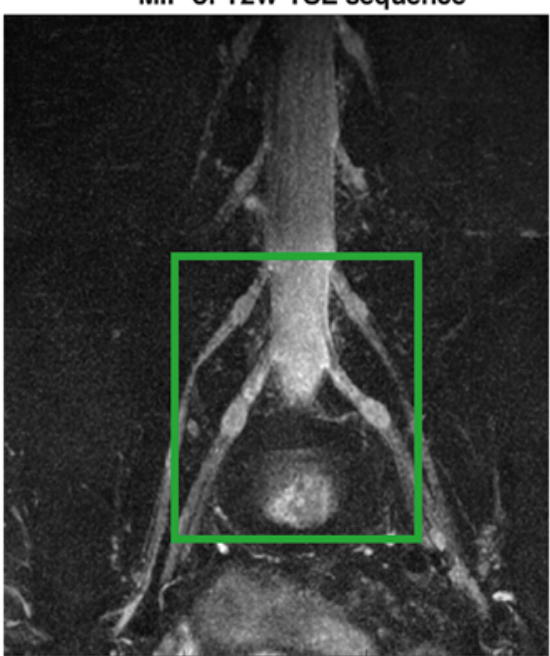

MIP of T2W TSE sequence

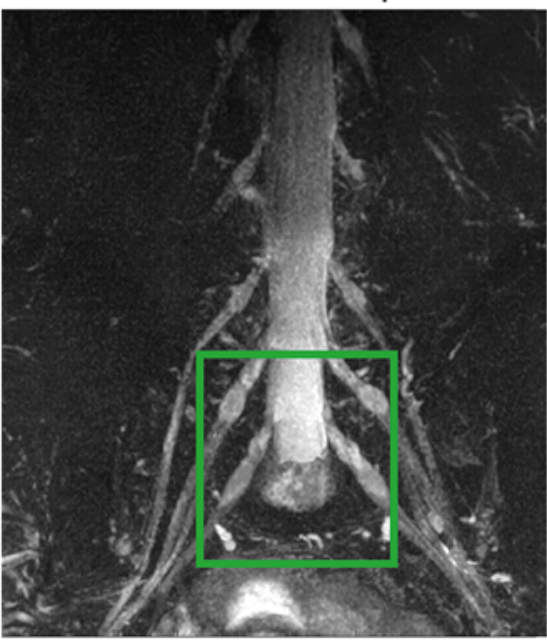

T2 map overlay (in ms)

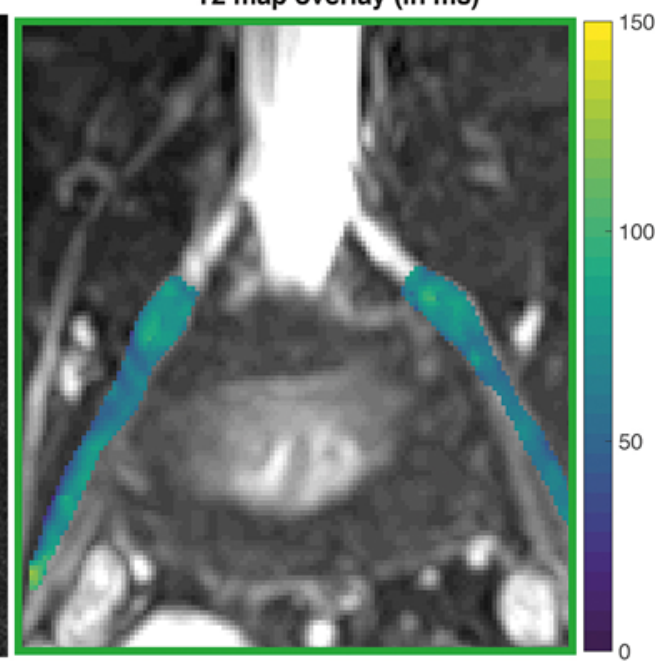

T2 map overlay (in ms)

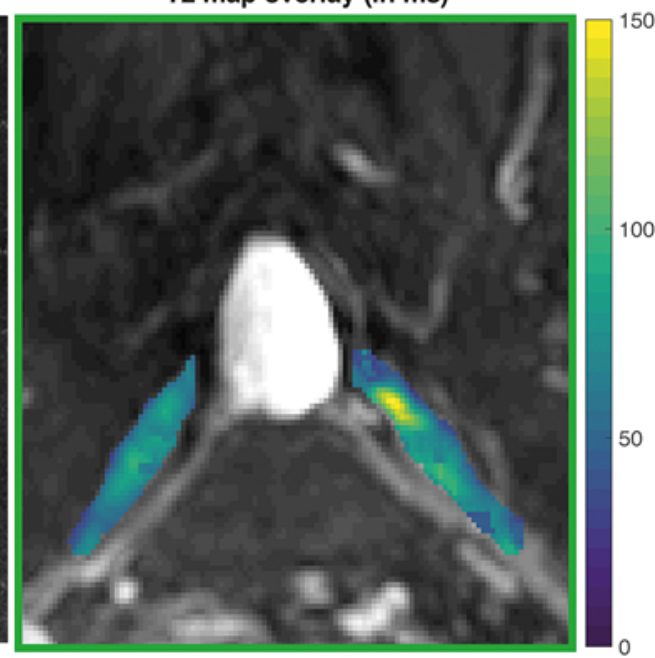

FIG. 1. This figure illustrates two representative cases: one without a side-related difference in T2 values (upper row) and the other with unilateral elevated T2 values according to T2 mapping (lower row) when considering the level of the suspected nerve in contact with degenerated disc material and causing symptoms. The figure first shows a sagittal overview of the lumbosacral spine (upper left and lower left) followed by coronal slices derived from the T2-weighted (T2W) 3D TSE sequence (maximum intensity projection [MIP]; upper center and lower center). Areas depicted in the green boxes are shown in the upper right and lower right panels; these images show T2 maps superimposed on coronal slices of the 3D TSE sequence, which were taken for extraction of T2 values (in msec [ms]) at preganglionic, ganglionic, and postganglionic sites at the level where the nerve is thought to make contact with degenerative disc material and cause symptoms. Both patients suffered from unilateral LRS that matched at least one dermatome according to clinical examination. Figure is available in color online only.

20/40/60/80 msec; TR 1.6 seconds; and TE of the TSE shot 15 msec. $^{25,37}$

A modified B1-insensitive rotation pulse was applied for $\mathrm{T} 2$ preparation in order to minimize the sensitivity to $\mathrm{B} 0$ and B1 inhomogeneities. ${ }^{25,37}$ The same scanning protocol using the same MRI scanner has been applied previously in healthy subjects. ${ }^{37}$ The duration of the T2 mapping sequence was 6 minutes 48 seconds.

\section{Data Analysis}

A voxel-by-voxel approach with a combination of golden section search and variable projection was used to generate T2 maps. ${ }^{18,24,37}$ The obtained maps were uploaded to Horos software (version 1.1.7; https://www.horosproject. org), which was used for placement of regions of interests (ROIs) and extraction of T2 values. The steps of postprocessing, including calculation of T2 values and ROI placement, were analogously performed previously in healthy individuals. ${ }^{37}$

During analysis, the investigator (with experience in neuroradiological imaging since 2012) was blinded to the side of symptoms but was informed about the level of suspected nerve contact with degenerated disc material according to previous anatomical MRI. Then, the level of the affected nerve was identified on the T2 maps with an applied color scheme, which did not allow for structural diagnosis of disc herniation (Fig. 1). The respective map was partly averaged (isotropic voxel size of $3 \mathrm{~mm}$ ) and 


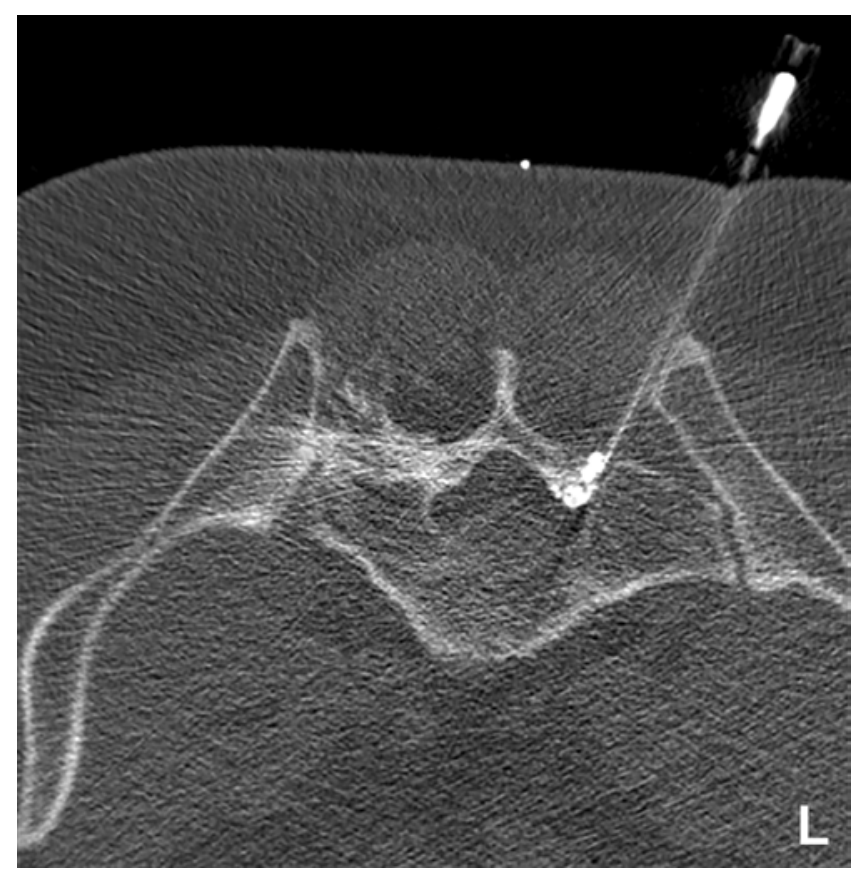

FIG. 2. This image shows an example of the approach used for CTguided ESI in a representative case of a patient suffering from left-sided LRS corresponding to the S1 dermatome. After contrast medium was applied, corticosteroid solution and local anesthetics were delivered.

manually reformatted to display a maximum length of the nerve course captured within the respective FOV (Fig. 1). Manual placement of polygonal ROIs in the axial slices of the T2 maps was then performed for the left and right nerves at the affected level at preganglionic (approximately $1 \mathrm{~cm}$ before the ganglion), ganglionic (in the middle of the ganglion), and postganglionic (approximately $1 \mathrm{~cm}$ after the ganglion) sites, and T2 values of these ROIs were subsequently extracted. This approach had shown high reproducibility in a previous study. ${ }^{37}$

As an internal reference, the L2 nerve on both sides was subsequently identified, and preganglionic, ganglionic, and postganglionic ROIs were drawn analogous to the approach on the affected level. Thus, 12 measurements of $\mathrm{T} 2$ values were performed in each patient (preganglionic, ganglionic, and postganglionic T2 values at the affected nerve level of both sides, and preganglionic, ganglionic, and postganglionic T2 values belonging to L2 of both sides). The preganglionic, ganglionic, and postganglionic measurements were averaged in each patient for each level and side, respectively, thus leading to 4 average T2 values for each patient.

After ROI placements and T2 value extractions from the T2 maps with applied color schemes, the T2-weighted 3D TSE sequences were evaluated to detect any qualitative signal alterations of the affected nerve. This was achieved by careful visual inspection of the images.

\section{ESI}

ESI was performed in each patient on the same or next day during clinical routine after MRN by experienced neuroradiologists while using CT monitoring (Brilliance
64, Philips Healthcare). The procedure was carried out with the patient in a prone position, starting with a first short planning CT scan that included the target level with the adjacent vertebral bodies. T2 maps were not available during injection planning or guidance.

Following sterile preparation, draping, and administration of local anesthesia, a spinal puncture needle $(0.9 \times 88$ $\mathrm{mm}$; Spinocan, Braun AG) was advanced stepwise using a paramedian technique to the target nerve root at the site where it exits the respective neural foramen (Fig. 2) ${ }^{1,3,35}$ After the correct approach and location of the needle were confirmed by repeated CT scans, contrast medium (Solutrast $250 \mathrm{M}$; Bracco Imaging $\mathrm{GmbH}$ ) was applied to assess correct local dispersion along the course of the nerve during a final CT scan (Fig. 2). Subsequently, $10 \mathrm{mg}$ of corticosteroid solution (Triamcinolon; Hexal AG) and 1 $\mathrm{ml}$ of a local anesthetic (Scandicain 2\%; AstraZeneca Plc) were delivered. Each patient was asked to again rate overall radicular pain according to the VAS, which was administered during a follow-up telephone call the day after the procedure.

\section{Statistical Analysis}

All statistical data analyses and generation of graphs were performed using GraphPad Prism (version 6.0; GraphPad Software Inc.) or SPSS (version 23.0; IBM Corp.). A p value $<0.05$ was defined as statistically significant.

The average $\mathrm{T} 2$ values of the affected nerve level on both sides were first grouped in measurements from the affected nerve and the contralateral nerve. For these measurements, those derived from the L2 nerve on both sides (as the control level) and patient characteristics, descriptive statistics, including absolute or relative frequencies, mean $\pm \mathrm{SD}$, median, minimum, and maximum, were calculated. The Shapiro-Wilk normality test indicated normal data distribution for the obtained $\mathrm{T} 2$ values.

We then compared the T2 values of the affected nerve against the contralateral nerve by using paired t-tests. The right- and left-sided T2 values of the L2 nerve as the control level were analogously compared against each other using paired t-tests.

Furthermore, after these data evaluations, we decided to define the following criteria for $\mathrm{T} 2$ values and responses to CT-guided ESI: aberrant T2 value (an aberrant T2 value for the affected nerve was defined as a value being outside of the range of the mean $\pm 2 \times$ SD derived from the contralateral nerve at the same level when considering the measurements of the whole cohort), and missing positive response to ESI (a change of the VAS score of $<$ $50 \%$ when comparing the individual VAS scores before and after ESI).

To be able to calculate receiver operating characteristics (ROCs) for the comparison of T2 values and responsiveness to ESI, we defined true positive (TP), true negative (TN), false positive (FP), and false negative (FN) fractions as follows:

- TP: aberrant T2 value AND positive response to ESI

- TN: nonaberrant T2 value AND missing positive response to ESI 
TABLE 1. Summary of patient characteristics

\begin{tabular}{|c|c|}
\hline Variable & Value (range) \\
\hline Median ODI score & $41.0 \%(14 \%-74 \%)$ \\
\hline \multicolumn{2}{|l|}{ Previous lumbar decompression } \\
\hline Yes & $16.7 \%$ \\
\hline No & $83.3 \%$ \\
\hline Median time since last lumbar op, yrs & $29.8(18-44)$ \\
\hline Median duration of Sx, wks & $10.0(4-24)$ \\
\hline \multicolumn{2}{|l|}{ Side of Sx } \\
\hline Right & $38.9 \%$ \\
\hline Left & $61.1 \%$ \\
\hline \multicolumn{2}{|l|}{ Affected nerve* } \\
\hline L3 & $5.6 \%$ \\
\hline L4 & $22.2 \%$ \\
\hline L5 & $61.1 \%$ \\
\hline S1 & $11.1 \%$ \\
\hline \multicolumn{2}{|l|}{ LE paresthesia/hypesthesia } \\
\hline Yes & $33.3 \%$ \\
\hline No & $76.7 \%$ \\
\hline \multicolumn{2}{|l|}{ LE motor status $\dagger$} \\
\hline $5 / 5$ & $100 \%$ \\
\hline$<5 / 5$ & $0 \%$ \\
\hline \multicolumn{2}{|l|}{ Median VAS score } \\
\hline Before ESI & $7.0(5-10)$ \\
\hline After ESI & $2.5(0-7)$ \\
\hline \multicolumn{2}{|l|}{ Later lumbar decompression } \\
\hline Yes & $33.3 \%$ \\
\hline No & $67.7 \%$ \\
\hline
\end{tabular}

LE = lower-extremity; ODI = Oswestry Disability Index; Sx = symptoms. Values are presented as the percentage of patients or the median (range).

${ }^{*}$ According to clinical examination and anatomical imaging.

$\dagger$ According to the BMRC scale.

- FP: aberrant T2 value AND missing positive response to ESI, and

- FN: nonaberrant T2 value AND positive response to ESI.

Furthermore, we also compared T2 values to qualitative, visually assessed signal alterations in the T2-weighted 3D TSE sequences (TP: aberrant T2 value AND clear T2 signal increases; TN: nonaberrant T2 value AND no clear T2 signal increases; FP: aberrant T2 value AND no clear T2 signal increases; and FN: nonaberrant T2 value AND clear T2 signal increases) and to the clinical status (TP: aberrant T2 value AND clinical symptoms with strict unilateral LRS; TN: nonaberrant T2 value AND no clinical symptoms with strict unilateral LRS; FP: aberrant T2 value AND no clinical symptoms with strict unilateral LRS; and FN: nonaberrant T2 value AND clinical symptoms with strict unilateral LRS) within the context of further ROC analyses.

\section{Results}

Between April 2016 and October 2017, 18 patients (10
TABLE 2. T2 values derived from measurements at the affected and control levels

\begin{tabular}{lcccc}
\hline & \multicolumn{4}{c}{ Measurement Site (msec) } \\
\cline { 2 - 5 } Nerve & Preganglionic & Ganglionic & Postganglionic & Overall \\
\hline $\begin{array}{c}\text { Affected } \\
\text { nerve }\end{array}$ & $76.4 \pm 2.2$ & $87.3 \pm 2.7$ & $68.0 \pm 1.5$ & $77.3 \pm 1.9$ \\
\hline $\begin{array}{c}\text { Contralat } \\
\text { nerve }\end{array}$ & $74.1 \pm 1.7$ & $84.3 \pm 2.1$ & $65.9 \pm 1.7$ & $74.8 \pm 1.4$ \\
\hline p value & 0.0001 & 0.0001 & $<0.0001$ & $<0.0001$ \\
\hline Left L2 & $72.5 \pm 1.1$ & $82.9 \pm 1.0$ & $64.6 \pm 1.3$ & $73.3 \pm 0.9$ \\
\hline Right L2 & $72.7 \pm 1.4$ & $82.8 \pm 1.0$ & $64.8 \pm 1.3$ & $73.4 \pm 1.0$ \\
\hline p value & 0.0634 & 0.4996 & 0.0827 & 0.2667 \\
\hline
\end{tabular}

An overall value is given per nerve, which represents the average derived from these 3 segments. Measurement values are presented in milliseconds as the mean $\pm \mathrm{SD}$.

males and 8 females; mean age $64.4 \pm 10.2$ years), all presenting with strict unilateral LRS, were included. Three of the patients presented with additional LBP (16.7\%). Previous anatomical MRI indicated disc herniation due to degeneration with unilateral nerve contact in all cases. MRN, including analyses of T2 maps, was carried out successfully in all patients without technical or methodological problems (Fig. 1). Cohort characteristics are shown in Table 1.

For affected nerves, the mean T2 was $77.3 \pm 1.9 \mathrm{msec}$, whereas it accounted for $74.8 \pm 1.4 \mathrm{msec}$ for the contralateral nerves ( $p<0.0001$; Table 2 and Fig. 3). Measurements along the left $\mathrm{L} 2$ nerve resulted in a mean T2 value of 73.3 $\pm 0.9 \mathrm{msec}$, whereas that for the right L2 nerve was $73.4 \pm$ $1.0 \mathrm{msec}(\mathrm{p}=0.2667$; Table 2$)$. This level was regarded as an internal control level and, thus, did not show related LRS and was not affected by structural pathology according to anatomical MRI (Table 1). Furthermore, signal alterations shown on the T2-weighted 3D TSE sequences according to visual inspection were present within the affected nerves in 4 patients (22.2\%), whereas the remaining 14 patients (78.8\%) did not show any clear T2 signal alterations.

Aberrant T2 values were found in 12 patients (66.7\%), and 13 patients $(72.2 \%)$ demonstrated a positive response to ESI. When comparing T2 values and the responsiveness to ESI, 10 patients (55.6\%) fulfilled the criteria for $\mathrm{TP}$, whereas 3 patients $(16.7 \%)$ fulfilled the criteria for $\mathrm{TN}$. Another 2 patients $(11.0 \%)$ were categorized as FP and 3 patients (16.7\%) as FN. Accordingly, T2 mapping showed a sensitivity/specificity of $76.9 \% / 60.0 \%$ and a positive predictive value (PPV)/negative predictive value (NPV) of $83.3 \% / 50.0 \%$ in relation to ESI.

When considering signal alterations in the T2-weighted 3D TSE sequences according to visual inspection, T2 mapping demonstrated a sensitivity/specificity of $100.0 \% / 42.9 \%$ and a PPV/NPV of 33.3\%/100.0\%. In relationship to clinical symptoms with all included patients suffering from strict unilateral LRS, T2 mapping showed a sensitivity of $66.7 \%$ and a PPV of $100.0 \%$.

\section{Discussion}

This study aimed to detect nerve affection due to disc 
herniation by means of high-resolution MRN in combination with T2 mapping of LSP nerves in patients with unilateral LRS. We observed higher T2 values in the affected nerves and observed an association between $\mathrm{T} 2$ values and the individual response to ESI with local anesthetics performed after MRN.

In a clinical routine, patients suffering from severe or longer-lasting LRS commonly undergo anatomical imaging as one of the first diagnostic steps with the aim of detecting structural pathologies as possible causes for the condition. However, although anatomical MRI, in particular, is capable of detecting degenerative disc disease as a major cause of LRS, the situation becomes complicated because it has been shown repeatedly that imaging findings do not necessarily correlate with clinical symptoms. In this context, there is a considerably high rate of anatomical examinations in which herniated discs are shown in asymptomatic patients, or symptomatic patients present without herniated discs. Specifically, herniated discs have been diagnosed in about $20 \%-36 \%$ of asymptomatic subjects, and symptoms resolved without decompressive surgery and without detectable herniation volume decreases in a considerable number of patients. ${ }^{12,20,31}$ Hence, the clear attribution of clinical symptoms to degenerative disc disease affecting nerves, but also the attribution of symptoms to an exact site, particularly in patients with multisegmental degenerative changes, is difficult in a considerable percentage of patients when solely relying on anatomical imaging. As such, CT or MRI, commonly used for anatomical imaging in the routine clinical setting, is primarily applied for the evaluation of surrounding structures possibly compressing the nerve that is clinically suspected to be the cause of the LRS, but these modalities do not routinely allow for the specific assessment of the nerve itself. Thus, other imaging approaches might be highly needed, which would ideally be able to provide objective information about the nerve suspected as the cause of symptoms.

Against this background, MRN is increasingly applied for the delineation of peripheral nerve pathologies related to inflammatory, neoplastic, metabolic, traumatic, or degenerative conditions. ${ }^{7-9,27}$ However, mostly qualitative MRN evaluations are performed with a focus on assessing signal alterations in T2-weighted imaging, which can be altered by such pathologies. . $10,11,21,36$ Thus, to date, T2 mapping that can provide detailed and more objective quantitative assessment is not routinely performed in patients with LRS, although it has shown to be feasible in healthy individuals at the level of the LSP. ${ }^{31,38}$ Furthermore, T2 mapping has also recently been successfully performed in patients with diabetic peripheral neuropathy: T2 values of the tibial nerve, originating from the LSP, have been shown to be clearly elevated in patients suffering from neuropathy compared to patients without neuropathy as well as healthy controls. $^{44}$

However, the LSP is known to be particularly challenging for both qualitative and quantitative MRN approaches due to the complex geometry of the nerves exiting the spinal canal, which require high isotropic resolution. Sufficient resolution has been shown to be achievable with advanced 3D TSE or 3D fast spin echo combined with improved motion-sensitized driven-equilibrium preparation; $4,10,11,21,36$

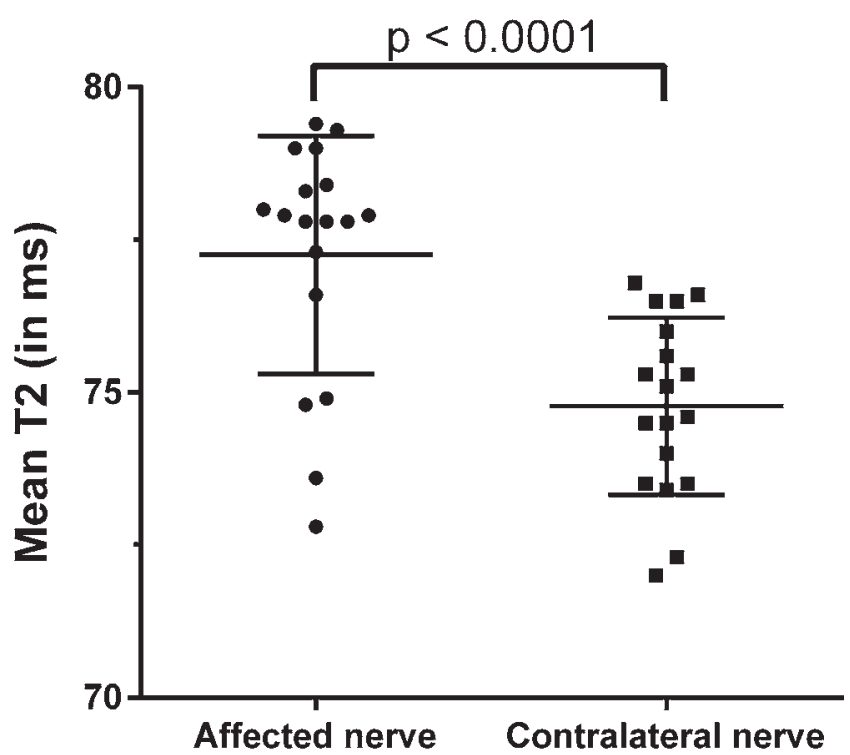

FIG. 3. T2 values derived from measurements at the affected level. The plots show the T2 values (in $\mathrm{msec}$ ) of each patient for the affected and contralateral unaffected nerve. Horizontal lines represent the mean with the SD. A statistically significant difference was observed $(p<0.0001)$, with clearly higher $\mathrm{T} 2$ values for the affected nerves.

however, evaluation can be significantly affected by B0 and B1 inhomogeneities that are regularly present in the LSP area. The T2-prepared 3D TSE sequence used in the present study has been demonstrated to enable B1-insensitive T2 quantification with high reproducibility and sufficient interobserver reliability, thus enabling T2 mapping of LSP nerves. ${ }^{37}$ However, the sequence has not yet been applied in patients with herniated discs and nerve contact, although the potential diagnostic usefulness seems evident.

Our current approach in such patients showed a statistically significant difference between affected and contralateral nerves, with higher T2 values for the affected nerves compared to the contralateral nerves (Table 2 and Fig. 3). This was observed in a cohort of patients who all presented with unilateral LRS but with uncertainty regarding the distinct level of the affected nerve causing symptoms because diagnostic ESI was scheduled in all of these patients. Furthermore, studies in only $22.2 \%$ of patients showed signal alterations within the affected nerves according to visual inspection of the T2-weighted 3D TSE sequences. For the nonaffected L2 nerve bilaterally, regarded as the control nerve, the difference in $\mathrm{T} 2$ values between the sides was not statistically significant (Table 2). Thus, our approach demonstrates that an aberration in T2 values is most probably due to the compressive effects of herniated disc material, as shown by high-resolution and B1-insensitive 3D MRN.

Furthermore, we calculated the ROC based on the responsiveness of patients to CT-guided ESI performed at the level corresponding to the symptoms according to clinical examination and previous anatomical MRI. Evaluation showed a sensitivity/specificity of $76.9 \% / 60.0 \%$, with 13 cases $(72.2 \%)$ being categorized as either TP or TN. In these patients, a good accordance between responsiveness to ESI and the distribution of T2 values has been shown, 
with the TN fraction probably being related to the finding that a different nerve than the clinically suspected one might be the main cause of the symptoms. In this context, evaluation of $\mathrm{T} 2$ values at additional levels that might also have been affected was not performed, making it possible that a different nerve level was primarily causing symptoms, which were wrongly attributed to the nerve level focused on during ESI and T2 mapping. Furthermore, 5 patients $(28.8 \%)$ were categorized as FP or FN, indicating inconclusive results when comparing the responsiveness to ESI and the distribution of T2 values. In this context, ESI has been shown to lead to only a significant reduction in pain intensity in about $60 \%-80 \%$ of patients, on average. ${ }^{2,23,32,38}$ Thus, although aberrant $\mathrm{T} 2$ values are present in the FP fraction, ESI might have failed to relieve symptoms due to nonresponsiveness of selected cases. Furthermore, the FN fraction could have shown a positive response to ESI due to placebo effects, which can occur in a considerable proportion of patients. ${ }^{33,39}$ Some patients exhibiting the FN fraction may have also experienced spreading of the local anesthetic into the epidural space, possibly resulting in distribution to adjacent nerve levels after the procedure; consequently, the positive response to ESI might have been the result of an additional effect of the injection on a cranially or caudally located nerve root. Future studies are needed to further explore the FP and FN fractions.

\section{Study Limitations}

There are limitations to this study that need to be taken into account when interpreting the data. First, to evaluate ROC, comparison of T2 mapping results to ESI was performed, with ESI being, clinically, the most widely available and most commonly applied diagnostic tool in addition to anatomical imaging for selected cases. However, further comparison of results to electromyography or nerve-conduction studies might be helpful to elucidate whether T2 mapping or ESI has provided more conclusive results in the FP and FN fractions. Comparisons between qualitative MRN and electromyography or nerve-conduction studies have already been achieved in patients with different nerve pathologies, demonstrating promising overall concordance. ${ }^{15}$ Second, a small fraction of our patients $(16.7 \%)$ suffered from LBP in addition to unilateral LRS. This could have potentially confounded our results because LBP might be regarded as a multifactorial condition, including that it might affect multilevel nerve roots bilaterally. Thus, future studies may exclusively focus on patients presenting with unilateral LRS and without LBP. Third, we used T2 mapping without the additional use of diffusionweighted imaging, which, however, can help during identification of nerve segments and fiber courses. Fourth, the applied T2 preparation did not involve diffusion or flowencoding gradients, making the sequence potentially susceptible to signal contamination by surrounding fluid and vessels. Thus, more advanced $\mathrm{T} 2$ preparation modules with diffusion and flow-encoding gradients should be applied to eliminate such contamination..$^{5,21,45}$ Fifth, T2 measurements could principally be hampered by the T2-weighted signal intensity's dependency on the orientation of the nerve in relation to the magnetic field (so-called magic-angle effect); however, the incorporation of $\mathrm{T} 2$ changes due to the magic-angle effect is considered to be minor when the axis of a respective nerve forms a small angle with the main magnetic field. ${ }^{6,22}$

\section{Conclusions}

This study used high-resolution MRN with T2 mapping of LSP nerves in patients suffering from unilateral LRS related to disc herniation; we found that the affected nerves were characterized by elevated $\mathrm{T} 2$ values compared with the contralateral nerves, whereas an unaffected control level did not show any significant side-related differences in T2 values. This finding was revealed in patients who did not regularly exhibit qualitative signal alterations in nerves; thus, quantitative MRN by means of T2 mapping could be a diagnostically useful add-on tool to anatomical MRI in neurosurgical patients, showing an acceptable sensitivity/specificity of $76.9 \% / 60.0 \%$ in relation to ESI with local anesthetics.

\section{References}

1. Benzon HT: Epidural steroid injections for low back pain and lumbosacral radiculopathy. Pain 24:277-295, 1986

2. Bonetti M, Fontana A, Cotticelli B, Volta GD, Guindani M, Leonardi M: Intraforaminal $\mathrm{O}_{2}-\mathrm{O}_{3}$ versus periradicular steroidal infiltrations in lower back pain: randomized controlled study. AJNR Am J Neuroradiol 26:996-1000, 2005

3. Cannon DT, Aprill CN: Lumbosacral epidural steroid injections. Arch Phys Med Rehabil 81 (3 Suppl 1):S87-S100, 2000

4. Cervantes B, Bauer JS, Zibold F, Kooijman H, Settles M, Haase A, et al: Imaging of the lumbar plexus: optimized refocusing flip angle train design for 3D TSE. J Magn Reson Imaging 43:789-799, 2016

5. Cervantes B, Kirschke JS, Klupp E, Kooijman H, Börnert P, Haase A, et al: Orthogonally combined motion- and diffusion-sensitized driven equilibrium (OC-MDSDE) preparation for vessel signal suppression in 3D turbo spin echo imaging of peripheral nerves in the extremities. Magn Reson Med 79:407-415, 2018

6. Chappell KE, Robson MD, Stonebridge-Foster A, Glover A, Allsop JM, Williams AD, et al: Magic angle effects in MR neurography. AJNR Am J Neuroradiol 25:431-440, 2004

7. Chhabra A, Andreisek G, Soldatos T, Wang KC, Flammang AJ, Belzberg AJ, et al: MR neurography: past, present, and future. AJR Am J Roentgenol 197:583-591, 2011

8. Chhabra A, Belzberg AJ, Rosson GD, Thawait GK, Chalian M, Farahani SJ, et al: Impact of high resolution 3 tesla MR neurography (MRN) on diagnostic thinking and therapeutic patient management. Eur Radiol 26:1235-1244, 2016

9. Chhabra A, Madhuranthakam AJ, Andreisek G: Magnetic resonance neurography: current perspectives and literature review. Eur Radiol 28:698-707, 2018

10. Chhabra A, Thawait GK, Soldatos T, Thakkar RS, Del Grande F, Chalian M, et al: High-resolution 3T MR neurography of the brachial plexus and its branches, with emphasis on 3D imaging. AJNR Am J Neuroradiol 34:486-497, 2013

11. Cho Sims G, Boothe E, Joodi R, Chhabra A: 3D MR neurography of the lumbosacral plexus: obtaining optimal images for selective longitudinal nerve depiction. AJNR Am J Neuroradiol 37:2158-2162, 2016

12. Delauche-Cavallier MC, Budet C, Laredo JD, Debie B, Wybier M, Dorfmann H, et al: Lumbar disc herniation. Computed tomography scan changes after conservative treatment of 
nerve root compression. Spine (Phila Pa 1976) 17:927-933, 1992

13. Deyo RA, Mirza SK, Martin BI: Back pain prevalence and visit rates: estimates from U.S. national surveys, 2002. Spine (Phila Pa 1976) 31:2724-2727, 2006

14. Dionne CE, Dunn KM, Croft PR, Nachemson AL, Buchbinder R, Walker BF, et al: A consensus approach toward the standardization of back pain definitions for use in prevalence studies. Spine (Phila Pa 1976) 33:95-103, 2008

15. Du R, Auguste KI, Chin CT, Engstrom JW, Weinstein PR: Magnetic resonance neurography for the evaluation of peripheral nerve, brachial plexus, and nerve root disorders. J Neurosurg 112:362-371, 2010

16. Fairbank J: Use of Oswestry Disability Index (ODI). Spine (Phila Pa 1976) 20:1535-1537, 1995

17. Fairbank JC, Pynsent PB: The Oswestry Disability Index. Spine (Phila Pa 1976) 25:2940-2952, 2000

18. Golub GH, Pereyra V: Differentiation of pseudo-inverses and nonlinear least-squares problems whose variables separate. SIAM J Numer Anal 10:413-432, 1973

19. Hart LG, Deyo RA, Cherkin DC: Physician office visits for low back pain. Frequency, clinical evaluation, and treatment patterns from a U.S. national survey. Spine (Phila Pa 1976) 20:11-19, 1995

20. Jensen MC, Brant-Zawadzki MN, Obuchowski N, Modic MT, Malkasian D, Ross JS: Magnetic resonance imaging of the lumbar spine in people without back pain. N Engl J Med 331:69-73, 1994

21. Kasper JM, Wadhwa V, Scott KM, Rozen S, Xi Y, Chhabra A: SHINKEI-a novel 3D isotropic MR neurography technique: technical advantages over 3DIRTSE-based imaging. Eur Radiol 25:1672-1677, 2015

22. Kästel T, Heiland S, Bäumer P, Bartsch AJ, Bendszus M, Pham M: Magic angle effect: a relevant artifact in MR neurography at 3T? AJNR Am J Neuroradiol 32:821-827, 2011

23. Kelekis A, Filippiadis DK, Velonakis G, Martin JB, Oikonomopoulos N, Brountzos E, et al: Fluoroscopically guided infiltration of the cervical nerve root: an indirect approach through the ipsilateral facet joint. Pain Physician 17:291296, 2014

24. Kiefer J: Sequential minimax search for a maximum. Proc Am Math Soc 4:502-506, 1953

25. Klupp E, Weidlich D, Schlaeger S, Baum T, Cervantes B, Deschauer M, et al: B1-insensitive T2 mapping of healthy thigh muscles using a T2-prepared 3D TSE sequence. PLoS One 12:e0171337, 2017

26. Koes BW, van Tulder MW, Peul WC: Diagnosis and treatment of sciatica. BMJ 334:1313-1317, 2007

27. Kollmer J, Bendszus M, Pham M: MR neurography: diagnostic imaging in the PNS. Clin Neuroradiol 25 (Suppl 2):283-289, 2015

28. Konstantinou K, Dunn KM: Sciatica: review of epidemiological studies and prevalence estimates. Spine (Phila Pa 1976) 33:2464-2472, 2008

29. Kronlage M, Schwehr V, Schwarz D, Godel T, Heiland S, Bendszus M, et al: Magnetic resonance neurography: normal values and demographic determinants of nerve caliber and T2 relaxometry in 60 healthy individuals. Clin Neuroradiol [epub ahead of print], 2017

30. Langley GB, Sheppeard H: The visual analogue scale: its use in pain measurement. Rheumatol Int 5:145-148, 1985

31. Maigne JY, Rime B, Deligne B: Computed tomographic follow-up study of forty-eight cases of nonoperatively treated lumbar intervertebral disc herniation. Spine (Phila Pa 1976) 17:1071-1074, 1992

32. Manchikanti L, Pampati V, Benyamin RM, Boswell MV: Analysis of efficacy differences between caudal and lumbar interlaminar epidural injections in chronic lumbar axial discogenic pain: local anesthetic alone vs. local combined with steroids. Int J Med Sci 12:214-222, 2015
33. Nandi J, Chowdhery A: A randomized controlled clinical trial to determine the effectiveness of caudal epidural steroid injection in lumbosacral sciatica. J Clin Diagn Res 11:RC04-RC08, 2017

34. Price DD, McGrath PA, Rafii A, Buckingham B: The validation of visual analogue scales as ratio scale measures for chronic and experimental pain. Pain 17:45-56, 1983

35. Shamliyan TA, Staal JB, Goldmann D, Sands-Lincoln M: Epidural steroid injections for radicular lumbosacral pain: a systematic review. Phys Med Rehabil Clin N Am 25:471489, 489.e1-489.e50, 2014

36. Soldatos T, Andreisek G, Thawait GK, Guggenberger R, Williams EH, Carrino JA, et al: High-resolution 3-T MR neurography of the lumbosacral plexus. Radiographics 33:967-987, 2013

37. Sollmann N, Weidlich D, Cervantes B, Klupp E, Ganter C, Kooijman $\mathrm{H}$, et al: High isotropic resolution T2 mapping of the lumbosacral plexus with T2-prepared 3D turbo spin echo. Clin Neuroradiol [epub ahead of print], 2018

38. Taskaynatan MA, Tezel K, Yavuz F, Tan AK: The effectiveness of transforaminal epidural steroid injection in patients with radicular low back pain due to lumbar disc herniation two years after treatment. J Back Musculoskeletal Rehabil 28:447-451, 2015

39. Valat JP: Epidural corticosteroid injections for sciatica: placebo effect, injection effect or anti-inflammatory effect? Nat Clin Pract Rheumatol 2:518-519, 2006

40. Van Boxem K, Cheng J, Patijn J, van Kleef M, Lataster A, Mekhail N, et al: 11. Lumbosacral radicular pain. Pain Pract 10:339-358, 2010

41. Vos T, Flaxman AD, Naghavi M, Lozano R, Michaud C, Ezzati M, et al: Years lived with disability (YLDs) for 1160 sequelae of 289 diseases and injuries 1990-2010: a systematic analysis for the Global Burden of Disease Study 2010. Lancet 380:2163-2196, 2012 (Erratum in Lancet 381:628, 2013)

42. Waggershauser T, Schwarzkopf S, Reiser M: [Facet blockade, peridural and periradicular pain therapy.] Radiologe 46:520526, 2006 (Ger)

43. Walker BF: The prevalence of low back pain: a systematic review of the literature from 1966 to 1998. J Spinal Disord 13:205-217, 2000

44. Wang D, Wang C, Duan X, Yang Z, Bai Z, Hu H, et al: MR T2 value of the tibial nerve can be used as a potential noninvasive and quantitative biomarker for the diagnosis of diabetic peripheral neuropathy. Eur Radiol 28:1234-1241, 2018

45. Yoneyama M, Takahara T, Kwee TC, Nakamura M, Tabuchi T: Rapid high resolution MR neurography with a diffusionweighted pre-pulse. Magn Reson Med Sci 12:111-119, 2013

\section{Disclosures}

The study was financed by institutional grants from the Department of Diagnostic and Interventional Radiology and the Department of Diagnostic and Interventional Neuroradiology. This project was furthermore partially supported by Philips Healthcare and has received funding from the European Research Council (ERC) under the European Union's Horizon 2020 research and innovation program (grant agreement no. 637164-iBack-ERC2014-STG).

H.K. is an employee of Philips Healthcare. J.S.K. received speaker honoraria from Philips Healthcare. D.C.K. receives grant support from Philips Healthcare. B.M. received honoraria, consulting fees, and research grants from Medtronic, Icotec ag, and Relievant Medsystems Inc.; honoraria and research grants from Ulrich Medical; honoraria and consulting fees from Spineart Deutschland $\mathrm{GmbH}$ and DePuy Synthes; consulting fees from Brainlab 
AG and Ulrich Medical; and royalties from Spineart Deutschland $\mathrm{GmbH}$.

\section{Author Contributions}

Conception and design: Sollmann, Weidlich, Cervantes, Klupp, Kirschke, Karampinos. Acquisition of data: Sollmann, Weidlich, Cervantes, Klupp, Kirschke, Karampinos. Analysis and interpretation of data: Sollmann, Weidlich, Cervantes, Klupp, Baum, Kirschke, Karampinos. Drafting the article: Sollmann, Weidlich, Cervantes, Karampinos. Critically revising the article: all authors. Reviewed submitted version of manuscript: all authors. Approved the final version of the manuscript on behalf of all authors:
Sollmann. Statistical analysis: Sollmann, Weidlich, Cervantes, Baum, Karampinos. Administrative/technical/material support: Sollmann, Ganter, Kooijman, Zimmer, Rummeny, Meyer, Baum, Kirschke, Karampinos. Study supervision: Zimmer, Rummeny, Meyer, Baum, Kirschke, Karampinos.

\section{Correspondence}

Nico Sollmann: Klinikum rechts der Isar, Technische Universität München, Munich, Germany.nico.sollmann@tum.de. 